\title{
A Modified Adomian Decomposition Method for Solving Higher-Order Singular Boundary Value Problems
}

\author{
Weonbae Kim ${ }^{\mathrm{a}}$ and Changbum Chun ${ }^{\mathrm{b}}$

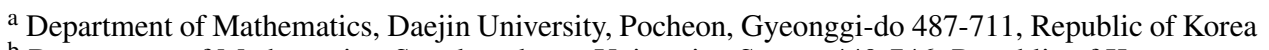 \\ b Department of Mathematics, Sungkyunkwan University, Suwon 440-746, Republic of Korea \\ Reprint requests to C. C.; Fax: +82-31-290-7033, E-mail: cbchun@skku.edu \\ Z. Naturforsch. 65a, 1093 - 1100 (2010); received August 24, 2009 / revised April 19, 2010

\begin{abstract}
In this paper, we present a reliable modification of the Adomian decomposition method for solving presented method, revealing its reliability and applicability in handling the problems with singular nature.
\end{abstract} \\ higher-order singular boundary value problems. He's polynomials are also used to overcome the com- \\ plex and difficult calculation of Adomian polynomials occurring in the application of the Adomian \\ decomposition method. Numerical examples are given to illustrate the accuracy and efficiency of the
}

Key words: Adomian Decomposition Method; Singular Boundary Value Problems;

Higher-Order Ordinary Differential Equation; Modified Adomian Decomposition Method;

He's polynomials; Homotopy Perturbation Method.

PACS number: $02.60 . \mathrm{Lj}$

\section{Introduction}

The Adomian decomposition method (ADM) is one of the powerful and reliable methods for solving various kinds of problems arising in applied sciences, linear and nonlinear as well. This method, which accurately computes the series solution, is of great interest to applied sciences $[1,2]$. The method provides the solution in a rapidly convergent series solution, if the equation has a unique solution, and it has been successfully applied to a wide class of problems arising in applied sciences, see [1-6], and the references therein. The convergence of the decomposition series have been investigated by several authors [7-9]. In recent years, the Adomian method has been modified so as to solve boundary value problems with singular nature by several authors [10-13]. The difficulty of those singular problems is due to the singularity behaviour that occurs at the point $x=0$.

In this paper we introduce a new reliable modification of the ADM to overcome the singularity difficulty for higher-order boundary value problems. He's polynomials with the homotopy perturbation method (HPM) are also used to overcome the complex and difficult calculation of Adomian polynomials occurring in the application of the Adomian decom- position method, demonstrating that He's polynomials can completely replace Adomian's polynomials. The HPM, first proposed by $\mathrm{He}$ in 1998, was developed and improved by $\mathrm{He}$ in [14-16]. This method is a novel and effective method [17-19], and has been successfully applied to solve many types of problems. For a comprehensive survey on the method, new interpretation, and its applications, the reader is referred to $[17,18]$. Several numerical examples will be considered to illustrate that the proposed framework is well suited to attain an accurate solution to the higher-order singular boundary value problems, revealing its reliability and applicability.

\section{Modified Adomian Decomposition Method}

Consider the singular boundary value problem of $(n+1)$-order ordinary differential equation in the form

$$
\begin{aligned}
& y^{(n+1)}+\frac{m}{x} y^{(n)}+N y=g(x), \\
& \begin{aligned}
y(0) & =a_{0}, y^{\prime}(0)=a_{1}, \ldots, y^{(r-1)}(0) \\
& =a_{r-1}, y(b)=c_{0}, y^{\prime}(b)=c_{1}, \ldots,
\end{aligned} \\
& y^{(n-r)}(b)=c_{n-r},
\end{aligned}
$$


where $N$ is a nonlinear differential operator of order less than $n, g(x)$ is a given function, $a_{0}, a_{1}, \ldots, a_{r-1}$, $c_{0}, c_{1}, \ldots, c_{n-r}, b$ are given constants, where $m \leq r \leq$ $n, r \geq 1$.

We first rewrite (1) in the form

$$
\begin{aligned}
x^{-2} \frac{\mathrm{d}^{n-1}}{\mathrm{~d} x^{n-1}}\left[x^{2} y^{\prime \prime}\right. & +(m-2 n+2) x y^{\prime} \\
& +(n-m)(n-1) y]+N y=g(x),
\end{aligned}
$$

or equivalently,

$$
\begin{aligned}
& x^{-2} \frac{\mathrm{d}^{n-1}}{\mathrm{~d} x^{n-1}}\left[x^{2 n-m} \frac{\mathrm{d}}{\mathrm{d} x}\left(x^{m-2 n+2} \frac{\mathrm{d} y}{\mathrm{~d} x}\right)\right] \\
& +(n-m)(n-1) x^{-2} \frac{\mathrm{d}^{n-1} y}{\mathrm{~d} x^{n-1}}+N y=g(x) .
\end{aligned}
$$

Equation (4) can be written in the operator form

$$
L_{2} L_{1} y=g(x)+(m-n)(n-1) L_{2} y-N y,
$$

where the differential operator $L$ employs the first two derivatives

$$
\begin{aligned}
& L_{1}=x^{2 n-m} \frac{\mathrm{d}}{\mathrm{d} x}\left(x^{m-2 n+2} \frac{\mathrm{d}}{\mathrm{d} x}\right), \\
& L_{2}=x^{-2} \frac{\mathrm{d}^{n-1}}{\mathrm{~d} x^{n-1}}
\end{aligned}
$$

in order to overcome the singularity behaviour at $x=0$.

In view of (6) and (7), the inverse operators $L_{1}^{-1}$ and $L_{2}^{-1}$ are the integral operators defined by

$$
\begin{aligned}
& L_{1}^{-1}(.)=\int_{b}^{x} x^{2 n-m-2} \int_{0}^{x} x^{m-2 n}(.) \mathrm{d} x \mathrm{~d} x \\
& L_{2}^{-1}(.)=\underbrace{\int_{0}^{x} \cdots \int_{0}^{x}}_{n-1 \text { times }} x^{2}(.) \mathrm{d} x \cdots \mathrm{d} x .
\end{aligned}
$$

By applying $L_{2}^{-1}$ on (5), we have

$$
L_{1} y=\Psi_{1}(x)+L_{2}^{-1} g(x)-L_{2}^{-1} N y,
$$

such that

$$
L_{2} \Psi_{1}(x)=0 .
$$

By applying $L_{1}^{-1}$ on (10), we have

$$
\begin{aligned}
y(x)= & \Psi_{2}(x)+L_{1}^{-1} \Psi_{1}(x)+L_{1}^{-1} L_{2}^{-1} g(x) \\
& -L_{1}^{-1} L_{2}^{-1} N y,
\end{aligned}
$$

such that

$$
L_{1} \Psi_{2}(x)=0 .
$$

The Adomian decomposition method introduces the solution $y(x)$ and the nonlinear function $N y$ by infinite series

$$
y(x)=\sum_{n=0}^{\infty} y_{n}(x)
$$

and

$$
N y=\sum_{n=0}^{\infty} A_{n}\left(y_{0}, y_{1}, \ldots, y_{n}\right),
$$

where the Adomian polynomials $A_{n}$ are defined as

$$
A_{n}=\left[\frac{1}{n !} \frac{\mathrm{d}^{n}}{\mathrm{~d} \lambda^{n}} N\left(\sum_{i=0}^{n} y_{i}(\lambda)^{i}\right)\right]_{\lambda=0} .
$$

The components $y_{n}(x)$ of the solution $y(x)$ will be determined recurrently, and Adomian polynomials can be constructed for various classes of nonlinearity according to specific algorithms presented in [6] and [20].

Substituting (14) and (15) into (12) gives

$$
\begin{aligned}
\sum_{n=0}^{\infty} y_{n}= & \Psi_{2}(x)+L_{1}^{-1} \Psi_{1}(x)+L_{1}^{-1} L_{2}^{-1} g(x) \\
& -L_{1}^{-1} L_{2}^{-1} \sum_{n=0}^{\infty} A_{n} .
\end{aligned}
$$

Identifying $y_{0}=\Psi_{2}(x)+L_{1}^{-1} \Psi_{1}(x)+L_{1}^{-1} L_{2}^{-1} g(x)$, the Adomian method admits the use of the recursive relation

$$
\begin{aligned}
& y_{0}=\Psi_{2}(x)+L_{1}^{-1} \Psi_{1}(x)+L_{1}^{-1} L_{2}^{-1} g(x), \\
& y_{n+1}=-L^{-1} A_{n}, \quad n \geq 0,
\end{aligned}
$$

which gives

$$
\begin{aligned}
& y_{0}=\Psi(x)+L^{-1} g(x), \\
& y_{1}=-L^{-1} A_{0}, \\
& y_{2}=-L^{-1} A_{1}, \\
& y_{3}=-L^{-1} A_{2},
\end{aligned}
$$

This will lead to the complete determination of the components $y_{n}(x)$ of $y(x)$. The series solution of $y(x)$ 
defined by (14) follows immediately. For numerical purposes, the $n$-term approximant

$$
\Psi_{n}=\sum_{k=0}^{n-1} y_{k}
$$

can be used to approximate the exact solution.

\section{Numerical Examples}

Example 1. Consider the nonlinear boundary value problem

$$
\begin{aligned}
& y^{\prime \prime}-\frac{1}{x} y^{\prime}=\frac{x^{2}}{3} y^{5}, \\
& y(0)=1, \quad y^{\prime}(1)=-\frac{\sqrt{3}}{8} .
\end{aligned}
$$

We define

$$
L_{1}=x^{3} \frac{\mathrm{d}}{\mathrm{d} x}\left(x^{-1} \frac{\mathrm{d}}{\mathrm{d} x}\right)
$$

so that

$$
L_{1}^{-1}(.)=\int_{0}^{x} x \int_{1}^{x} x^{-3}(.) \mathrm{d} x \mathrm{~d} x
$$

After multiplying (21) by $x^{2}$, in an operator form, it becomes

$$
L_{1} y=\frac{x^{4}}{3} y^{5}
$$

Applying $L_{1}^{-1}$ on both sides of (25) we find

$$
y(x)=y(0)+\frac{1}{2} y^{\prime}(1) x^{2}+L_{1}^{-1} \frac{x^{4}}{3} y^{5} .
$$

Using the decomposition series for the linear function $y(x)$ and the polynomial series for the nonlinear term $y^{5}$, we obtain

$$
\sum_{n=0}^{\infty} y_{n}=y(0)+\frac{1}{2} y^{\prime}(1) x^{2}+L_{1}^{-1}\left(\frac{x^{4}}{3} \sum_{n=0}^{\infty} A_{n}\right) .
$$

This gives the recursive relationship

$$
\begin{aligned}
& y_{0}(x)=y(0)+\frac{1}{2} y^{\prime}(1) x^{2}, \\
& y_{k+1}(x)=L_{1}^{-1}\left(\frac{x^{4}}{3} A_{k}\right), \quad k \geq 0 .
\end{aligned}
$$

The Adomian polynomials for the nonlinear term $y^{5}$ are computed as follows:

$$
\begin{aligned}
& A_{0}=y_{0}^{5}, \\
& A_{1}=5 y_{0}^{4} y_{1}, \\
& A_{2}=5 y_{2} y_{0}^{4}+10 y_{0}^{3} y_{1}^{2}, \\
& A_{3}=5 y_{3} y_{0}^{4}+20 y_{2} y_{1} y_{0}^{3}+10 y_{1}^{3} y_{0}^{2},
\end{aligned}
$$

Substituting (30) into (29) gives the components

$$
\begin{aligned}
y_{0}= & 1-0.108253 x^{2} \\
y_{1}= & -0.0637827 x^{2}+0.0416666 x^{4} \\
& -0.0075176 x^{6}+0.00081380 x^{8} \\
& -0.00005286 x^{10}+1.9073486 \cdot 10^{-6} x^{12} \\
& -2.9496649 \cdot 10^{-8} x^{14}+\cdots \\
y_{2}= & 0.0062577 x^{2}-0.0044294 x^{6} \\
& +0.0024057 x^{8}-0.0006259 x^{10} \\
& +0.0001017 x^{12}-0.0000114 x^{14}+\cdots, \\
y_{3}= & -0.0010668 x^{2}+0.0004346 x^{6} \\
& +0.0001884 x^{8}-0.0003596 x^{10} \\
& +0.0001864 x^{12}-0.0000553 x^{14}+\cdots,
\end{aligned}
$$

The solution in a series form is thus approximately given by

$$
\begin{aligned}
y= & y_{0}+y_{1}+y_{2}+y_{3} \\
= & 1-0.1668450 x^{2}+0.0416667 x^{4} \\
& -0.0115124 x^{6}+0.0034079 x^{8} \\
& -0.0010384 x^{10}+0.0002900 x^{12} \\
& -0.0000668 x^{14}+\cdots,
\end{aligned}
$$

which is in good agreement with the Taylor series of the exact solution $y(x)=\frac{1}{\sqrt{1+\frac{x^{2}}{3}}}$ given by

$$
\begin{aligned}
y(x)= & 1-0.166667 x^{2}+0.0416667 x^{4} \\
& -0.0115741 x^{6}+0.00337577 x^{8} \\
& -0.0010123 x^{10}+0.0003094 x^{12} \\
& -0.0000958 x^{14}+\cdots .
\end{aligned}
$$

For the above and later computations, the Maple package was used and the digits environment variable was 
set to 20 as the number of digits that Maple uses when calculating with software floating-point numbers. The results rounded off to seven decimal places are presented for the sake of simplicity.

Example 2. Consider the nonlinear boundary value problem

$$
\begin{gathered}
y^{\prime \prime}+\frac{\rho}{x} y^{\prime}=g(x), \\
y(0)=0, y^{\prime}(b)=b^{-\rho}(1-\rho) \cos b-b^{1-\rho} \sin b,
\end{gathered}
$$

where

$$
g(x)=-x^{1-\rho} \cos x-(2-\rho) x^{-\rho} \sin x .
$$

We define

$$
\begin{aligned}
& L_{1}=x^{2-\rho} \frac{\mathrm{d}}{\mathrm{d} x}\left(x^{\rho} \frac{\mathrm{d}}{\mathrm{d} x}\right), \\
& L_{2}=x^{-2},
\end{aligned}
$$

so that

$$
\begin{aligned}
& L_{1}^{-1}(.)=\int_{0}^{x} x^{-\rho} \int_{b}^{x} x^{\rho-2}(.) \mathrm{d} x \mathrm{~d} x, \\
& L_{2}^{-1}(.)=x^{2}(.) .
\end{aligned}
$$

In an operator form, (34) becomes

$$
L_{2} L_{1} y=g(x) .
$$

We find that

$$
\begin{aligned}
L_{1}^{-1} L_{1} y & =\int_{0}^{x} x^{-\rho} \int_{b}^{x} x^{\rho-2}\left[x^{2-\rho} \frac{\mathrm{d}}{\mathrm{d} x}\left(x^{\rho} \frac{\mathrm{d} y}{\mathrm{~d} x}\right)\right] \mathrm{d} x \mathrm{~d} x \\
& =\int_{0}^{x} x^{-\rho}\left[x^{\rho} y^{\prime}-b^{\rho} y^{\prime}(b)\right] \mathrm{d} x \\
& =y(x)-y(0)-\frac{b^{\rho}}{1-\rho} y^{\prime}(b) x^{1-\rho}
\end{aligned}
$$

Applying $L_{2}^{-1}, L_{1}^{-1}$ on both sides of (41), we find after imposing the given boundary conditions

$$
\begin{aligned}
& y(x)=y(0)+\frac{b^{\rho}}{1-\rho} y^{\prime}(b) x^{1-\rho} \\
& +\int_{0}^{x} x^{-\rho} \int_{b}^{x}[-x \cos x-(2-\rho) \sin x] \mathrm{d} x \mathrm{~d} x= \\
& \frac{1}{1-\rho}[(1-\rho) \cos b-b \sin b] x^{1-\rho} \\
& +[\cos x-\cos b] x^{1-\rho}+\frac{b \sin b}{1-\rho} x^{1-\rho}=x^{1-\rho} \cos x,
\end{aligned}
$$

which is the exact solution of the problem (34) and (35).
Example 3. Consider the nonlinear boundary value problem

$$
\begin{aligned}
& y^{\prime \prime \prime}+\frac{3}{x} y^{\prime \prime}-y^{3}=g(x), \\
& y(0)=0, \quad y^{\prime}(0)=0, \quad y(1)=\mathrm{e},
\end{aligned}
$$

where $g(x)=24 \mathrm{e}^{x}+36 x \mathrm{e}^{x}+12 x^{2} \mathrm{e}^{x}+x^{3} \mathrm{e}^{x}-x^{9} \mathrm{e}^{3 x}$. We use the Taylor series of $g(x)$ with order 10 ,

$$
\begin{aligned}
& g(x) \approx g_{T}=24+60 x+60 x^{2}+35 x^{3}+14 x^{4}+\frac{21}{5} x^{5} \\
& +x^{6}+\frac{11}{56} x^{7}+\frac{11}{336} x^{8}-\frac{30097}{30240} x^{9}-\frac{64787}{21600} x^{10} .
\end{aligned}
$$

We define

$$
\begin{aligned}
& L_{1}=x \frac{\mathrm{d}}{\mathrm{d} x}\left(x \frac{\mathrm{d}}{\mathrm{d} x}\right), \\
& L_{2}=x^{-2} \frac{\mathrm{d}}{\mathrm{d} x},
\end{aligned}
$$

so that

$$
\begin{aligned}
& L_{1}^{-1}(.)=\int_{0}^{x} x^{-1} \int_{0}^{x} x^{-1}(.) \mathrm{d} x \mathrm{~d} x . \\
& L_{2}^{-1}(.)=\int_{0}^{x} x^{2}(.) \mathrm{d} x .
\end{aligned}
$$

In an operator form, (44) becomes

$$
L_{2} L_{1} y=g_{T}(x)+L_{2} y+y^{3} .
$$

Applying $L_{2}^{-1}, L_{1}^{-1}$ on both sides of (51) and then incorporating the given boundary conditions, we find

$$
y(x)=L_{1}^{-1} L_{2}^{-1} g_{T}(x)+L_{1}^{-1} y+L_{1}^{-1} L_{2}^{-1} y^{3} .
$$

Using the decomposition series for the linear function $y(x)$ and the polynomial series for the nonlinear term $y^{3}$, we obtain

$$
\begin{aligned}
\sum_{n=0}^{\infty} y_{n}= & L_{1}^{-1} L_{2}^{-1} g_{T}(x)+L_{1}^{-1}\left(\sum_{n=0}^{\infty} y_{n}\right) \\
& +L_{1}^{-1} L_{2}^{-1}\left(\sum_{n=0}^{\infty} A_{n}\right) .
\end{aligned}
$$

This gives the recursive relationship

$$
\begin{aligned}
& y_{0}(x)=L_{1}^{-1} L_{2}^{-1} g_{T}(x), \\
& y_{k+1}(x)=L_{1}^{-1} y_{k}+L_{1}^{-1} L_{2}^{-1} A_{k}, \quad k \geq 0 .
\end{aligned}
$$


The Adomian polynomials for the nonlinear term $y^{3}$ are computed as follows:

$$
\begin{aligned}
& A_{0}=y_{0}^{3}, \\
& A_{1}=3 y_{0}^{2} y_{1}, \\
& A_{2}=3 y_{2} y_{0}^{2}+3 y_{0} y_{1}^{2}, \\
& A_{3}=3 y_{3} y_{0}^{2}+6 y_{0} y_{1} y_{2}+y_{1}^{3},
\end{aligned}
$$

Substituting (56) into (55) gives the components of the solution

$$
\begin{aligned}
y_{0}= & 0.8888889 x^{3}+0.9375000 x^{4}+0.4800000 x^{5} \\
& +0.1620370 x^{6}+0.0408163 x^{7}+0.0082031 x^{8} \\
& +0.0013717 x^{9}+0.0001964 x^{10}+0.0000246 x^{11} \\
& -0.0005759 x^{12}-0.0013652 x^{13}, \\
y_{1}= & 0.0987654 x^{3}+0.0585938 x^{4}+0.0192000 x^{5} \\
& +0.0045010 x^{6}+0.0008329 x^{7}+0.0001282 x^{8} \\
& +0.0000169 x^{9}+0.0000019 x^{10}+0.0000002 x^{11} \\
& +\cdots, \\
y_{2}= & 0.0109739 x^{3}+0.0036621 x^{4}+0.0007680 x^{5} \\
& +0.0001250 x^{6}+0.0000169 x^{7}+0.0000020 x^{8} \\
& +2.0907516 \times 10^{-7} x^{9}+1.9642857 \times 10^{-8} x^{10} \\
& +1.6799819 \times 10^{-9} x^{11}+\cdots, \\
y_{3}= & 0.0012193 x^{3}+0.0002289 x^{4}+0.0000307 x^{5} \\
& +0.00000347 x^{6}+3.4693305 \times 10^{-7} x^{7} \\
& +3.1292439 \times 10^{-8} x^{8}+2.5811748 \times 10^{-9} x^{9} \\
& +1.9642857 \times 10^{-10} x^{10}+1.3884148 \times 10^{-11} x^{11}, \\
& +\cdots,
\end{aligned}
$$

The solution in a series form is thus approximately given by

$$
\begin{aligned}
y= & y_{0}+y_{1}+y_{2}+y_{3} \\
= & 0.9998476 x^{3}+0.9999847 x^{4}+0.4999987 x^{5} \\
& +0.16666666 x^{6}+0.0416667 x^{7}+0.0083333 x^{8} \\
& +0.0013888 x^{9}+0.0001984 x^{10}+0.0000248 x^{11} \\
& \cdots,
\end{aligned}
$$

which is in good agreement with the Taylor series of the exact solution $y(x)=x^{3} \mathrm{e}^{x}$ given by

$$
\begin{aligned}
y(x)= & x^{3}+x^{4}+0.5 x^{5}+0.1666666 x^{6} \\
& +0.0416666 x^{7}+0.008333 x^{8} \\
& +0.0013888 x^{9}+0.000198 x^{10} \\
& +0.0000248 x^{11}+\cdots .
\end{aligned}
$$

Example 4. Consider the nonlinear boundary value problem

$$
\begin{aligned}
& y^{(4)}+\frac{3}{x} y^{(3)}+y^{2}-y^{3}=g(x), \\
& y(0)=0, y^{\prime}(0)=0, y(1)=\mathrm{e}, y^{\prime}(1)=3 \mathrm{e},
\end{aligned}
$$

where $g(x)=18 x^{-1} \mathrm{e}^{x}+30 \mathrm{e}^{x}+11 x \mathrm{e}^{x}+x^{2} \mathrm{e}^{x}+x^{4} \mathrm{e}^{2 x}$ $-x^{6} \mathrm{e}^{3 x}$. We use the 11 terms of the Taylor series of $g(x)$,

$$
\begin{aligned}
& g(x) \approx \frac{18}{x}+48+50 x+30 x^{2}+\frac{49}{4} x^{3}+\frac{71}{15} x^{4} \\
& +\frac{29}{10} x^{5}+\frac{33}{28} x^{6}-\frac{6599}{4032} x^{7}-\frac{9649}{2520} x^{8}-\frac{1279991}{302400} x^{9} .
\end{aligned}
$$

We define

$$
\begin{aligned}
& L_{1}=x^{3} \frac{\mathrm{d}}{\mathrm{d} x}\left(x^{-1} \frac{\mathrm{d}}{\mathrm{d} x}\right), \\
& L_{2}=x^{-2} \frac{\mathrm{d}^{2}}{\mathrm{~d} x^{2}},
\end{aligned}
$$

so that

$$
\begin{aligned}
& L_{1}^{-1}(.)=\int_{0}^{x} x \int_{1}^{x} x^{-3}(.) \mathrm{d} x \mathrm{~d} x, \\
& L_{2}^{-1}(.)=\int_{0}^{x} \int_{0}^{x} x^{2}(.) \mathrm{d} x \mathrm{~d} x .
\end{aligned}
$$

In an operator form, (60) then becomes

$$
L_{2} L_{1} y=g(x)+y^{3}-y^{2} .
$$

Applying $L_{2}^{-1}$ on both sides of (67) and then incorporating given boundary conditions, (60) becomes

$$
\begin{aligned}
L_{1} y= & \left.L_{1} y\right|_{x=0}+\left.\frac{\mathrm{d}}{\mathrm{d} x}\left(L_{1} y\right)\right|_{x=0} \cdot x \\
& +L_{2}^{-1} g(x)+L_{2}^{-1}\left(y^{3}-y^{2}\right) \\
= & -y^{\prime}(0) x+L_{2}^{-1} g(x)+L_{2}^{-1}\left(y^{3}-y^{2}\right) \\
= & L_{2}^{-1} g(x)+L_{2}^{-1}\left(y^{3}-y^{2}\right) .
\end{aligned}
$$


Since $L_{1}^{-1} L_{1} y=y(x)-y(0)-\frac{1}{2} y^{\prime}(1) x^{2}$, applying $L_{1}^{-1}$ on both sides of (68) with given boundary conditions yields

$$
\begin{aligned}
y(x)= & \frac{3 \mathrm{e}}{2} x^{2}+L_{1}^{-1} L_{2}^{-1} g(x) \\
& +L_{1}^{-1} L_{2}^{-1}\left(y^{3}-y^{2}\right) .
\end{aligned}
$$

Proceeding as before, we have the recursive relationship

$$
\begin{aligned}
& y_{0}(x)=\frac{3 \mathrm{e}}{2} x^{2}+L_{1}^{-1} L_{2}^{-1} g(x), \\
& y_{k+1}(x)=L_{1}^{-1} L_{2}^{-1} A_{k}-L_{1}^{-1} L_{2}^{-1} B_{k}, \quad k \geq 0 .
\end{aligned}
$$

The Adomian polynomials for the nonlinear terms $y^{3}$ and $y^{2}$ are computed as follows:

$$
\begin{aligned}
& A_{0}=y_{0}^{3}, \\
& A_{1}=3 y_{0}^{2} y_{1}, \\
& A_{2}=3 y_{2} y_{0}^{2}+3 y_{0} y_{1}^{2}, \\
& A_{3}=3 y_{3} y_{0}^{2}+6 y_{0} y_{1} y_{2}+y_{1}^{3},
\end{aligned}
$$

and

$$
\begin{aligned}
& B_{0}=y_{0}^{2}, \\
& B_{1}=2 y_{0} y_{1}, \\
& B_{2}=2 y_{2} y_{0}+y_{1}^{2}, \\
& B_{3}=2 y_{3} y_{0}+2 y_{1} y_{2},
\end{aligned}
$$

respectively.

Substituting (72) and (73) into (71) gives the components of the solution

$$
\begin{aligned}
y_{0}= & 0.9993606 x^{2}+x^{3}+0.5 x^{4}+0.1666667 x^{5} \\
& +0.0416667 x^{6}+0.0083333 x^{7}+0.0017609 x^{8} \\
& +0.0006393 x^{9}+0.0001637 x^{10}-0.0001503 x^{11} \\
& -0.0002417 x^{12}-0.0001897 x^{13}, \\
y_{1}= & -0.0007032 x^{2}-0.0003715 x^{8}-0.0004406 x^{9} \\
& -0.0001391 x^{10}+0.0001527 x^{11}+\cdots, \\
y_{2}= & 2.9469693 \times 10^{-6} x^{2}+5.228892996 \times 10^{-7} x^{8} \\
& +3.1005857 \times 10^{-7} x^{9}-1.9496233 \times 10^{-7} x^{10} \\
& -3.6567264 \times 10^{-7} x^{11}+\cdots, \\
y_{3}= & -1.2055039 \times 10^{-8} x^{2}-2.3752524 \times 10^{-9} x^{8} \\
& -1.2993692 \times 10^{-9} x^{9}+1.0229462 \times 10^{-9} x^{10} \\
& +1.6686604 \times 10^{-9} x^{11}+\cdots,
\end{aligned}
$$

We thus obtain an approximate solution given by

$$
\begin{aligned}
y= & y_{0}+y_{1}+y_{2}+y_{3} \\
= & 0.9986603 x^{2}+x^{3}+0.5 x^{4}+0.1666667 x^{5} \\
& +0.0416667 x^{6}+0.0083333 x^{7} \\
& +0.0013899 x^{8}+0.0001990 x^{9} \\
& +0.0000244 x^{10}+2.0591302 \times 10^{-6} x^{11} \\
& +\cdots
\end{aligned}
$$

which is in well agreement with Taylor series of the exact solution $y(x)=x^{2} \mathrm{e}^{x}$ given by

$$
\begin{aligned}
y(x)= & x^{2}+x^{3}+0.5 x^{4}+0.1666667 x^{5} \\
& +0.0416667 x^{6}+0.0083333 x^{7} \\
& +0.0013888 x^{8}+0.0001984 x^{9} \\
& +0.0000248 x^{10}+2.7557319 \times 10^{-6} x^{11} \\
& +\cdots
\end{aligned}
$$

\section{Homotopy Perturbation Method with He's Polynomials}

It should be noted that the calculation of Adomian's polynomials to deal with the nonlinear terms in Example 1, Example 3, and Example 4 is complex and difficult. This aspect is the demerit of the application of Adomian's decomposition method, though this method has been proved to be more powerful and efficient than some existing methods [21]. This difficulty may be easily overcome without using Adomian's polynomials when the so-called He's polynomials are used to solve the problem. We illustrate this in this section by revisiting and solving Example 1 and Example 3 by the homotopy perturbation method (HPM) with He's polynomials [22-24].

Revisited Example 1. Consider the nonlinear boundary value problem

$$
\begin{aligned}
& y^{\prime \prime}-\frac{1}{x} y^{\prime}=\frac{x^{2}}{3} y^{5}, \\
& y(0)=1, \quad y^{\prime}(1)=-\frac{\sqrt{3}}{8} .
\end{aligned}
$$

With equation (26), we construct the following homotopy:

$$
y(x)-y(0)-\frac{1}{2} y^{\prime}(1) x^{2}-p L_{1}^{-1} \frac{x^{4}}{3} N(y)=0,
$$


where $N(y)=y^{5}, p \in[0,1]$ is the embedding parameter, $L_{1}^{-1}(\cdot)$ is defined by $(24)$.

According to He's homotopy perturbation method $[14,15]$, we assume that the solution of (79) is

$$
y=y_{0}+p y_{1}+p^{2} y_{2}+\cdots \text {. }
$$

When $p \rightarrow 1$, (80) becomes the approximate solution of (77) and (78), i. e.,

$$
\lim _{p \rightarrow 1} y=y_{0}+y_{1}+y_{2}+\cdots
$$

is the exact solution of (77) and (78). The convergence of the series in (81) to the exact solution is discussed by $\mathrm{He}$ in $[14,15]$.

The nonlinear term $N(y)$ can be expressed in the form

$$
N(y)=H\left(y_{0}\right)+p H\left(y_{0}, y_{1}\right)+p^{2} H\left(y_{0}, y_{1}, y_{2}\right)+\cdots,
$$

where $H\left(y_{0}, \cdots, y_{n}\right)$ is called He's polynomial [22] defined by

$$
\begin{aligned}
& H\left(y_{0}, \cdots, y_{n}\right)=\frac{1}{n !} \frac{\mathrm{d}^{n}}{\mathrm{~d} p^{n}} N\left(\sum_{k=0}^{n} p^{k} y_{k}\right)_{p=0}, \\
& n=0,1,2,3 \cdots .
\end{aligned}
$$

Substituting (80) and (82) into (79), and collecting the coefficients of like powers of $p$, we have

$$
\begin{aligned}
& {\left[y_{0}-y(0)-\frac{1}{2} y^{\prime}(1) x^{2}\right] p^{0}+\left[y_{1}-L_{1}^{-1} \frac{x^{4}}{3} y_{0}^{5}\right] p^{1}} \\
& +\left[y_{2}-L_{1}^{-1} \frac{x^{4}}{3}\left(5 y_{0}^{4} y_{1}\right)\right] p^{2}+\left[y_{3}-L_{1}^{-1} \frac{x^{4}}{3}\left(5 y_{2} y_{0}^{4}\right.\right. \\
& \left.\left.+10 y_{0}^{3} y_{1}^{2}\right)\right] p^{3}+\left[y_{4}-L_{1}^{-1} \frac{x^{4}}{3}\left(5 y_{3} y_{0}^{4}+20 y_{2} y_{1} y_{0}^{3}\right.\right. \\
& \left.\left.+10 y_{1}^{3} y_{0}^{2}\right)\right] p^{4}+\cdots=0,
\end{aligned}
$$

from which we have simultaneously the approximate solution to (77) and (78) given in (32) and He's polynomials

$$
\begin{aligned}
& H\left(y_{0}\right)=y_{0}^{5}, \\
& H\left(y_{0}, y_{1}\right)=5 y_{0}^{4} y_{1}, \\
& H\left(y_{0}, y_{1}, y_{2}\right)=5 y_{2} y_{0}^{4}+10 y_{0}^{3} y_{1}^{2}, \\
& H\left(y_{0}, y_{1}, y_{2}, y_{3}\right)=5 y_{3} y_{0}^{4}+20 y_{2} y_{1} y_{0}^{3}+10 y_{1}^{3} y_{0}^{2},
\end{aligned}
$$

Revisited Example 3. Consider the nonlinear boundary value problem

$$
\begin{aligned}
& y^{\prime \prime \prime}+\frac{3}{x} y^{\prime \prime}-y^{3}=g(x), \\
& y(0)=0, \quad y^{\prime}(0)=0, \quad y(1)=\mathrm{e},
\end{aligned}
$$

where $g(x)=24 \mathrm{e}^{x}+36 x \mathrm{e}^{x}+12 x^{2} \mathrm{e}^{x}+x^{3} \mathrm{e}^{x}-x^{9} \mathrm{e}^{3 x}$.

With equation (52), we construct the following homotopy:

$y(x)-L_{1}^{-1} L_{2}^{-1} g_{T}(x)-L_{1}^{-1} y-p L_{1}^{-1} L_{2}^{-1} N(y)=0$,

where $N(y)=y^{3}, p \in[0,1]$ is the embedding parameter and, $g_{T}, L_{1}^{-1}(\cdot)$ and $L_{2}^{-1}(\cdot)$ are defined by (46), (49), and (50), respectively.

We assume that the solution of (88) is

$$
y=y_{0}+p y_{1}+p^{2} y_{2}+\cdots .
$$

The nonlinear term $N(y)$ can be expressed in the form

$$
N(y)=H\left(y_{0}\right)+p H\left(y_{0}, y_{1}\right)+p^{2} H\left(y_{0}, y_{1}, y_{2}\right)+\cdots,
$$

where $H\left(y_{0}, \cdots, y_{n}\right)$ is the He polynomial defined by (83).

Substituting (89) and (90) into (88), and collecting the coefficients of like powers of $p$, we have

$$
\begin{aligned}
& {\left[y_{0}-L_{1}^{-1} L_{2}^{-1} g_{T}\right] p^{0}+\left[y_{1}-L_{1}^{-1} y_{0}-L_{1}^{-1} L_{2}^{-1}\left(y_{0}^{3}\right)\right] p^{1}} \\
& +\left[y_{2}-L_{1}^{-1} y_{1}-L_{1}^{-1} L_{2}^{-1}\left(3 y_{0}^{2} y_{1}\right)\right] p^{2}+\left[y_{3}-L_{1}^{-1} y_{2}\right. \\
& \left.-L_{1}^{-1} L_{2}^{-1}\left(3 y_{0}^{2} y_{2}+3 y_{0} y_{1}^{2}\right)\right] p^{3}+\left[y_{4}-L_{1}^{-1} y_{3}\right. \\
& \left.-L_{1}^{-1} L_{2}^{-1}\left(3 y_{3} y_{0}^{2}+6 y_{0} y_{1} y_{2}+y_{1}^{3}\right)\right] p^{4}+\cdots=0
\end{aligned}
$$

from which we have simultaneously the approximate solution to (86) and (87) given in (58) and He's polynomials

$$
\begin{aligned}
& H\left(y_{0}\right)=y_{0}^{3}, \\
& H\left(y_{0}, y_{1}\right)=3 y_{0}^{2} y_{1}, \\
& H\left(y_{0}, y_{1}, y_{2}\right)=3 y_{0}^{2} y_{2}+3 y_{0} y_{1}^{2}, \\
& H\left(y_{0}, y_{1}, y_{2}, y_{3}\right)=3 y_{3} y_{0}^{2}+6 y_{0} y_{1} y_{2}+y_{1}^{3},
\end{aligned}
$$

He's polynomials were used in the above examples to overcome the complex and difficult calculation of the Adomian polynomials in the Adomian decomposition method. We calculated He's polynomials and the solutions simultaneously, and observed that He's 
polynomials can completely replace Adomian's polynomials. This result reveals the applicability and efficiency of the homotopy perturbation method with higher-order singular boundary value problems.

\section{Conclusion}

In this paper, we present a reliable modification of the Adomian decomposition method to solve linear and nonlinear problems with singular feature. It is demonstrated that the presented approach can be well suited to attain an accurate solution to the higher-order singular boundary value problems, linear and nonlinear as well. The difficulty of those singular problems, due to the existence of the singular point at $x=0$, is over-

[1] G. Adomian, Math. Comput. Modelling 13, 17 (1992).

[2] G. Adomian, Solving frontier problems of physics: The decomposition method, Kluwer, Boston, MA, 1994.

[3] A. M. Wazwaz, Appl. Math. Comput. 166, 638 (2005).

[4] E. Deeba, G. Dibeh, and G. Xie, Appl. Math. Comput. 128, 81 (2002).

[5] E. Babolian and J. Biazar, Appl. Math. Comput. 129, 339 (2002).

[6] A. M. Wazwaz, Partial differential equations and solitary waves theory, Springer, New York 2009.

[7] K. Abbaoui and Y. Cherruault, Comput. Math. Appl. 29, 103 (1995).

[8] Y. Cherruault, G. Adomian, K. Abbaoui, and R. Rach, Bio-Medical Computing 38, 89 (1995).

[9] M. M. Hosseini and H. Nasabzadeh, Appl. Math. Comput. 182, 536 (2006).

[10] A. M. Wazwaz, Appl. Math. Comput. 128, 45 (2002).

[11] A. M. Wazwaz, Appl. Math. Comput. 161, 543 (2005).

[12] Y. Q. Hasan and L. M. Zhu, Commun. Nonlinear Sci. Numer. Simul. 14, 2592 (2009). come in this contribution. He's polynomials are also used to overcome the complex and difficult calculation of Adomian's polynomials occurring in the application of Adomian's decomposition method, illustrating that He's polynomials can completely replace Adomian's polynomials. The other types of differential equations with singular feature can also be similarly handled by the proposed approach.

\section{Acknowledgements}

This work was supported by the Daejin University Research Grants in 2010. The authors would like to thank the referees for their useful comments and constructive suggestions which substantially improved the quality of this paper.

[13] M. M. Hosseini and M. Jafari, Commun. Nonlinear Sci. Numer. Simul. 14, 1952 (2009).

[14] J. H. He, Comput. Methods Appl. Mech. Eng. 178, 257 (1999).

[15] J. H. He, Int. J. Nonlinear Mech. 35, 37 (2000).

[16] J. H. He, Appl. Math. Comput. 135, 73 (2003).

[17] J. H. He, Int. J. Modern Phys. B 20, 1144 (2006).

[18] J. H. He, Int. J. Modern Phys. B 20, 2561 (2006).

[19] A. Yildirim and D. Agirseven, Int. J. Nonlinear Sci. Numer. Simul. 10, 235 (2009).

[20] A. M. Wazwaz, Appl. Math. Comput. 111, 53 (2000).

[21] H. Bulut, Nonlinear Sci. Lett. A 1, 161 (2010).

[22] A. Ghorbani and J. S. Nadjfi, Int. J. Nonlinear Sci. Numer. Simul. 8, 229 (2007).

[23] A. Ghorbani, Chaos, Solitons, and Fractals 39, 1486 (2009).

[24] S. T. Mohyun-Din, Math. Probl. Eng. 2009, 427516 (2009). 